\title{
Generalized second-order parametric optimality conditions in semiinfinite discrete minmax fractional programming and second order $(\mathcal{F}, \beta, \phi, \rho, \theta$, $m$ )-univexity
}

\author{
Ram U. Verma ${ }^{1, *}$ and G. J. Zalmai ${ }^{2}$ \\ ${ }^{1}$ Department of Mathematics, University of North Texas, USA \\ ${ }^{2}$ Department of Mathematics and Computer Science, Northern Michigan University, USA
}

(Received: 5 January 2016; Accepted: 30 January 2016)

\begin{abstract}
This paper deals with mainly establishing numerous sets of generalized second-order parametric sufficient optimality conditions for a semiinfinite discrete minmax fractional programming problem, while the results on semiinfinite discrete minmax fractional programming problem are achieved based on some partitioning schemes under various types of generalized second-order $(\mathcal{F}, \beta, \phi, \rho, \theta$, m)-univexity assumptions.
\end{abstract}

Key Words Semiinfinite discrete minmax fractional programming, Generalized second-order $(\mathcal{F}, \beta, \phi, \rho, \theta, \mathrm{m})$-univex functions, Generalized sufficient optimality conditions.

AMS Mathematics Subject Classification (2000): 90C26, 90C30, 90C32, 90C46, 90C47

DOI: $10.19139 /$ soic.v4i1.188

\section{Introduction and Preliminaries}

Here in this paper, we plan to establish several sets of generalized parametric sufficient optimality conditions under various generalized $(\mathcal{F}, \beta, \phi, \rho, \theta, m)$-univexity assumptions for the following semiinfinite discrete minmax fractional programming problem of the form:

$$
\text { (P) Minimize } \max _{1 \leq i \leq p} \frac{f_{i}(x)}{g_{i}(x)}
$$

subject to

$$
\begin{aligned}
G_{j}(x, t) \leq 0 & \text { for all } t \in T_{j}, \quad j \in \underline{q}, \\
H_{k}(x, s)=0 & \text { for all } s \in S_{k}, \quad k \in \underline{r}, \\
& x \in X,
\end{aligned}
$$

where $X$ is an open convex subset of $\mathbb{R}^{n}$, the $n$-dimensional Euclidean space, for each $j \in q \equiv\{1,2, \ldots, q\}$ and $k \in \underline{r}, T_{j}$ and $S_{k}$ are compact subsets of complete metric spaces, for each $i \in p, f_{i}$ and $g_{i}$ are real-valued functions defined on $\mathbb{R}^{n}$, for each $j \in q, x \rightarrow G_{j}(x, t)$ is a real-valued function defined on $\mathbb{R}^{n}$ for all $t \in T_{j}$, for each $k \in \underline{r}, x \rightarrow H_{k}(x, s)$ is a real-valued function defined on $\mathbb{R}^{n}$ for all $s \in S_{k}$, for each $j \in q$ and $k \in \underline{r}, t \rightarrow G_{j}(x, t)$ and $s \rightarrow H_{k}(x, s)$ are continuous real-valued functions defined, respectively, on $T_{j}$ and $\bar{S}_{k}$ for all $x \in \mathbb{R}^{n}$, and for each $i \in \underline{p}, g_{i}(x)>0$ for all $x$ satisfying the constraints of $(\mathrm{P})$.

*Correspondence to: Ram Verma (Email: verma99@msn.com)

ISSN 2310-5070 (online) ISSN 2311-004X (print)

Copyright (C) 2016 International Academic Press 
This communication deals with investigating primarily results on various second-order necessary and sufficient optimality conditions for several types of optimization problems for the semiinfinite discrete minmax fractional programming based on generalized $(\mathcal{F}, \beta, \phi, \rho, \theta, m)$-univexity assumptions, while problems for the discrete minmax fractional programming based on generalized $(\mathcal{F}, \beta, \phi, \rho, \theta, m)$-univexity assumptions were initiated in [4]. Here, we shall apply two types of partitioning schemes due to Mond and Weir [3] to the context of the new classes of generalized second-order uninvex functions to formulate and discuss numerous sets of generalized second-order sufficient optimality conditions for $(P)$. To the best of our knowledge, all the secondorder sufficient optimality results established in this paper are new in the area of semiinfinite discrete minmax fractional programming. In fact, it seems that results of this type have not yet appeared in any shape or form for any type of mathematical programming problems. The generalized optimality conditions established here are suitable to utilize in constructing some generalized second-order parametric duality models for $(P)$ and proving numerous weak, strong, and strict converse duality theorems.

Section 1 of this paper is devoted first to some introductory materials on the semiinfinite discrete fractional programming and related developments, and then recalling some basic definitions and auxiliary results which will be needed in the sequel. In Section 2, we state and prove various second-order parametric sufficient optimality results for $(P)$ using a variety of generalized $(\mathcal{F}, \beta, \phi, \rho, \theta, m)$-sounivexity assumptions along with some partitioning schemes. Finally, in Section 3 we summarize our main results and also point out some further research opportunities arising from certain modifications of the principal problem investigated in the present paper leading to the concluding remarks.

We also observe that all the optimality results obtained for $(P)$ are also applicable, when appropriately specialized, to the following three classes of problems with discrete max, fractional, and conventional objective functions, which are particular cases of $(P)$ :

$$
\begin{aligned}
& \underset{x \in \mathbb{F}}{\operatorname{Minimize}} \max _{1 \leq i \leq p} f_{i}(x) ; \\
& \underset{x \in \mathbb{F}}{\operatorname{Minimize}} \frac{f_{1}(x)}{g_{1}(x)} \\
& \underset{x \in \mathbb{F}}{\operatorname{Minimize}} f_{1}(x)
\end{aligned}
$$

where $\mathbb{F}$ (assumed to be nonempty) is the feasible set of $(P)$, that is,

$$
\mathbb{F}=\left\{x \in X: G_{j}(x) \leq 0, j \in \underline{q}, \quad H_{k}(x)=0, \quad k \in \underline{r}\right\} .
$$

We next introduce some new classes of generalized second-order univex functions, referred to as (strictly) $(\mathcal{F}, \beta, \phi, \rho, \theta, m)$-sounivex, (strictly) $(\mathcal{F}, \beta, \phi, \rho, \theta, m)$-pseudosounivex, and (prestrictly) $(\mathcal{F}, \beta, \phi, \rho, \theta, m)$ quasisounivex functions. They are further extensions of the classes of second-order (strictly) $(\phi, \eta, \rho, \theta, m)$ sonvex, (strictly) $(\phi, \eta, \rho, \theta, m)$-pseudosonvex, and (prestrictly) $(\phi, \eta, \rho, \theta, m)$-quasisonvex functions which were introduced recently in [4]. For brief accounts of the evolution of the generalized $\mathcal{F}$-convex and other related functions, the reader is referred to $[4,5]$. We abbreviate "second-order univex" as sounivex. Let $f: X \rightarrow \mathbb{R}$ be a twice differentiable function.

Definition 1.1. The function $f$ is said to be (strictly) $(\mathcal{F}, \beta, \phi, \rho, \theta, m)$-sounivex at $x^{*}$ if there exist functions $\beta: X \times X \rightarrow \mathbb{R}_{+} \equiv(0, \infty), \phi: \mathbb{R} \rightarrow \mathbb{R}, \rho: X \times X \rightarrow \mathbb{R}, \theta: X \times X \rightarrow \mathbb{R}^{n}$, a sublinear function $\mathcal{F}\left(x, x^{*} ; \cdot\right):$ $\mathbb{R}^{n} \rightarrow \mathbb{R}$, and a positive integer $m\left(m \geq 1\right.$ such that for each $x \in X\left(x \neq x^{*}\right)$ and $z \in \mathbb{R}^{n}$,

$$
\phi\left(f(x)-f\left(x^{*}\right)\right)(>) \geq \mathcal{F}\left(x, x^{*} ; \beta\left(x, x^{*}\right) \nabla f\left(x^{*}\right)\right)+\frac{1}{2}\left\langle z, \nabla^{2} f\left(x^{*}\right) z\right\rangle+\rho\left(x, x^{*}\right)\left\|\theta\left(x, x^{*}\right)\right\|^{m},
$$

where $\|\cdot\|$ is a norm on $\mathbb{R}^{n}$ and $\langle a, b\rangle$ is the inner product of the vectors $a$ and $b$.

The function $f$ is said to be (strictly) $(\mathcal{F}, \beta, \phi, \rho, \theta, m)$-sounivex on $X$ if it is (strictly) $(\mathcal{F}, \beta, \phi, \rho, \theta, m)$-sounivex at each $x^{*} \in X$. 
Definition 1.2. The function $f$ is said to be (strictly) $(\mathcal{F}, \beta, \phi, \rho, \theta, m)$-pseudosounivex at $x^{*}$ if there exist functions $\beta: X \times X \rightarrow \mathbb{R}_{+}, \phi: \mathbb{R} \rightarrow \mathbb{R}, \rho: X \times X \rightarrow \mathbb{R}, \theta: X \times X \rightarrow \mathbb{R}^{n}$, a sublinear function $\mathcal{F}\left(x, x^{*} ; \cdot\right): \mathbb{R}^{n} \rightarrow \mathbb{R}$, and a positive integer $m\left(m \geq 1\right.$ such that for each $x \in X\left(x \neq x^{*}\right)$ and $z \in \mathbb{R}^{n}$,

$$
\mathcal{F}\left(x, x^{*} ; \beta\left(x, x^{*}\right) \nabla f\left(x^{*}\right)\right)+\frac{1}{2}\left\langle z, \nabla^{2} f\left(x^{*}\right) z\right\rangle \geq-\rho\left(x, x^{*}\right)\left\|\theta\left(x, x^{*}\right)\right\|^{m}
$$

$$
\Rightarrow \phi\left(f(x)-f\left(x^{*}\right)\right)(>) \geq 0 .
$$

The function $f$ is said to be (strictly) $(\mathcal{F}, \beta, \phi, \rho, \theta, m)$-pseudosounivex on $X$ if it is (strictly) $(\mathcal{F}, \beta, \phi, \rho, \theta, m)$ pseudosounivex at each $x^{*} \in X$.

Definition 1.3. The function $f$ is said to be (prestrictly) $(\mathcal{F}, \beta, \phi, \rho, \theta, m)$-quasisounivex at $x^{*}$ if there exist functions $\beta: X \times X \rightarrow \mathbb{R}_{+}, \phi: \mathbb{R} \rightarrow \mathbb{R}, \rho: X \times X \rightarrow \mathbb{R}, \theta: X \times X \rightarrow \mathbb{R}^{n}$, a sublinear function $\mathcal{F}\left(x, x^{*} ; \cdot\right)$ : $\mathbb{R}^{n} \rightarrow \mathbb{R}$, and a positive integer $m\left(m \geq 1\right.$ such that for each $x \in X$ and $z \in \mathbb{R}^{n}$,

$$
\phi\left(f(x)-f\left(x^{*}\right)\right)(<) \leq 0
$$

$$
\Rightarrow \mathcal{F}\left(x, x^{*} ; \beta\left(x, x^{*}\right) \nabla f\left(x^{*}\right)\right)+\frac{1}{2}\left\langle z, \nabla^{2} f\left(x^{*}\right) z\right\rangle \leq-\rho\left(x, x^{*}\right)\left\|\theta\left(x, x^{*}\right)\right\|^{m} .
$$

The function $f$ is said to be (prestrictly) $(\mathcal{F}, \beta, \phi, \rho, \theta, m)$-quasisounivex on $X$ if it is (prestrictly) $(\mathcal{F}, \beta, \phi, \rho, \theta, m)$-quasisounivex at each $x^{*} \in X$.

From the above definitions it is clear that if $f$ is $(\mathcal{F}, \beta, \phi, \rho, \theta, m)$-sounivex at $x^{*}$, then it is both $(\mathcal{F}, \beta, \phi, \rho, \theta, m)$ pseudosounivex and $(\mathcal{F}, \beta, \phi, \rho, \theta, m)$-quasisounivex at $x^{*}$, if $f$ is $(\mathcal{F}, \beta, \phi, \rho, \theta, m)$-quasisounivex at $x^{*}$, then it is prestrictly $(\mathcal{F}, \beta, \phi, \rho, \theta, m)$-quasisounivex at $x^{*}$, and if $f$ is strictly $(\mathcal{F}, \beta, \phi, \rho, \theta, m)$-pseudosounivex at $x^{*}$, then it is $(\mathcal{F}, \beta, \phi, \rho, \theta, m)$-quasisounivex at $x^{*}$.

In the proofs of the duality theorems, sometimes it may be more convenient to use certain alternative but equivalent forms of the above definitions. These are obtained by considering the contrapositive statements. For example, $(\mathcal{F}, \beta, \phi, \rho, \theta, m)$-quasisounivexity can be defined in the following equivalent way:

The function $f$ is said to be $(\mathcal{F}, \beta, \phi, \rho, \theta, m)$-quasisounivex at $x^{*}$ if there exist functions $\beta: X \times X \rightarrow \mathbb{R}_{+}, \phi$ : $\mathbb{R} \rightarrow \mathbb{R}, \rho: X \times X \rightarrow \mathbb{R}, \theta: X \times X \rightarrow \mathbb{R}^{n}$, a sublinear function $\mathcal{F}\left(x, x^{*} ; \cdot\right): \mathbb{R}^{n} \rightarrow \mathbb{R}$, and a positive integer $m$ ( $m \geq 1$ such that for each $x \in X$ and $z \in \mathbb{R}^{n}$,

$$
\mathcal{F}\left(x, x^{*} ; \beta\left(x, x^{*}\right) \nabla f\left(x^{*}\right)\right)+\frac{1}{2}\left\langle z, \nabla^{2} f\left(x^{*}\right) z\right\rangle>-\rho\left(x, x^{*}\right)\left\|\theta\left(x, x^{*}\right)\right\|^{m}
$$

$$
\Rightarrow \phi\left(f(x)-f\left(x^{*}\right)\right)>0 .
$$

Note that the new classes of generalized convex functions specified in Definitions $1.1-1.3$ contain a variety of special cases that can easily be identified by appropriate choices of $\mathcal{F}, \beta, \phi, \rho, \theta$, and $m$. For example, if we let $\mathcal{F}\left(x, x^{*} ; \nabla f\left(x^{*}\right)\right)=\left\langle\nabla f\left(x^{*}\right), \eta\left(x, x^{*}\right)\right\rangle$ and $\beta\left(x, x^{*}\right) \equiv 1$, then we obtain the definitions of (strictly) $(\phi, \eta, \rho, \theta, m)$-sonvex, (strictly) $(\phi, \eta, \rho, \theta, m)$-pseudosonvex, and (prestrictly) $(\phi, \eta, \rho, \theta, m)$-quasisonvex functions introduced recently in [4].

We conclude this section by recalling a set of second-order parametric necessary optimality conditions for $(P)$. The form and features of this result will provide clear guidelines for formulating various sets of second-order parametric sufficient optimality conditions for $(P)$.

Theorem 1.1. [4] Let $x^{*} \in \mathbb{F}$ and $\lambda^{*}=\max _{1 \leq i \leq p} f_{i}\left(x^{*}\right) / g_{i}\left(x^{*}\right)$, for each $i \in p$, let $f_{i}$ and $g_{i}$ be twice continuously differentiable at $x^{*}$, for each $j \in q$, let the function $z \rightarrow G_{j}(z, t)$ be twice continuously differentiable at $x^{*}$ for all $t \in T_{j}$, and for each $k \in \underline{r}$, let the function $z \rightarrow H_{k}(z, s)$ be twice continuously differentiable at $x^{*}$ for all $s \in S_{k}$. If $x^{*}$ is an optimal solution of (P), if the second order generalized Abadie constraint qualification holds at $x^{*}$, and 
if for any critical direction y, the set cone

$$
\begin{aligned}
& \left\{\left(\nabla G_{j}\left(x^{*}, t\right),\left\langle y, \nabla^{2} G_{j}\left(x^{*}, t\right) y\right\rangle\right): t \in \hat{T}_{j}\left(x^{*}\right), j \in \underline{q}\right\} \\
+ & \operatorname{span}\left\{\left(\nabla H_{k}\left(x^{*}, s\right),\left\langle y, \nabla^{2} H_{k}\left(x^{*}, s\right) y\right\rangle\right): s \in S_{k}, k \in \underline{r}\right\}, \\
& \text { where } \hat{T}_{j}\left(x^{*}\right) \equiv\left\{t \in T_{j}: G_{j}\left(x^{*}, t\right)=0\right\},
\end{aligned}
$$

is closed, then there exist $u^{*} \in U \equiv\left\{u \in \mathbb{R}^{p}: u \geq 0, \sum_{i=1}^{p} u_{i}=1\right\}$ and integers $\nu_{0}^{*}$ and $\nu^{*}$, with $0 \leq \nu_{0}^{*} \leq$ $\nu^{*} \leq n+1$, such that there exist $\nu_{0}^{*}$ indices $j_{m}$, with $1 \leq j_{m} \leq q$, together with $\nu_{0}^{*}$ points $t^{m} \in \hat{T}_{j_{m}}\left(x^{*}\right), m \in$ $\underline{\nu_{0}^{*}}, \nu^{*}-\nu_{0}^{*}$ indices $k_{m}$, with $1 \leq k_{m} \leq r$, together with $\nu^{*}-\nu_{0}^{*}$ points $s^{m} \in S_{k_{m}}$ for $m \in \underline{\nu^{*}} \backslash \underline{\nu_{0}^{*}}$, and $\nu^{*}$ real numbers $v_{m}^{*}$, with $v_{m}^{*}>0$ for $m \in \underline{\nu_{0}^{*}}$, with the property that

$$
\begin{aligned}
& \quad \sum_{i=1}^{p} u_{i}^{*}\left[\nabla f_{i}\left(x^{*}\right)-\lambda^{*}\left(\nabla g_{i}\left(x^{*}\right)\right]+\sum_{m=1}^{\nu_{0}^{*}} v_{m}^{*}\left[\nabla G_{j_{m}}\left(x^{*}, t^{m}\right)\right.\right. \\
& +\sum_{m=\nu_{0}^{*}+1}^{\nu^{*}} v_{m}^{*} \nabla H_{k_{m}}\left(x^{*}, s^{m}\right)=0, \\
& \left\langle y,\left[\sum_{i=1}^{p} u_{i}^{*}\left[\nabla^{2} f_{i}\left(x^{*}\right)-\lambda^{*} \nabla^{2} g_{i}\left(x^{*}\right)\right]+\sum_{m=1}^{\nu_{0}^{*}} v_{m}^{*} \nabla^{2} G_{j_{m}}\left(x^{*}, t^{m}\right)\right.\right. \\
& \left.\left.+\sum_{m=\nu_{0}^{*}+1}^{\nu_{m}^{*}} v_{m}^{*} \nabla^{2} H_{k_{m}}\left(x^{*}, s^{m}\right)\right] y\right\rangle \geq 0, \\
& u_{i}^{*}\left[f_{i}\left(x^{*}\right)-\lambda^{*} g_{i}\left(x^{*}\right)\right]=0, \quad i \in \underline{p},
\end{aligned}
$$

where $\underline{\nu} \backslash \underline{\nu_{0}}$ is the complement of the set $\underline{\nu_{0}}$ relative to the set $\underline{\nu}$.

\section{Generalized Second-Order Sufficient Optimality Conditions}

In this section, we discuss several families of sufficient optimality results under various generalized $(\mathcal{F}, \beta, \phi, \rho, \theta, m)$-sounivexity hypotheses imposed on certain combinations of the problem functions. This is accomplished by employing a certain partitioning scheme which was originally proposed in [4] for the purpose of constructing generalized dual problems for nonlinear programming problems. For this we need some additional notations.

Let $\nu_{0}$ and $\nu$ be integers, with $1 \leq \nu_{0} \leq \nu \leq n+1$, and let $\left\{J_{0}, J_{1}, \ldots, J_{M}\right\}$ and $\left\{K_{0}, K_{1}, \ldots, K_{M}\right\}$ be partitions of the sets $\underline{\nu_{0}}$ and $\underline{\nu} \backslash \underline{\nu_{0}}$, respectively; thus, $J_{i} \subset \underline{\nu_{0}}$ for each $i \in \underline{M} \cup\{0\}, J_{i} \cap J_{j}=\emptyset$ for each $i, j \in \underline{M} \cup\{0\}$ with $i \neq j$, and $\bar{\cup}_{i=0}^{M} J_{i}=\underline{\nu_{0}}$. Obviously, similar properties hold for $\left\{K_{0}, K_{1}, \ldots, K_{M}\right\}$. Moreover, if $m_{1}$ and $m_{2}$ are the numbers of the partitioning sets of $\underline{\nu_{0}}$ and $\underline{\nu} \underline{\nu_{0}}$, respectively, then $M=\max \left\{m_{1}, m_{2}\right\}$ and $J_{i}=\emptyset$ or $K_{i}=\emptyset$ for $i>\min \left\{m_{1}, m_{2}\right\}$.

In addition, we use the real-valued functions $z \rightarrow \Phi_{i}(z, \lambda, v, \bar{t}, \bar{s}), i \in \underline{p}, z \rightarrow \Phi(z, \lambda, u, v, \bar{t}, \bar{s})$, and $z \rightarrow$ $\Lambda_{\tau}(z, v, \bar{t}, \bar{s})$ defined, for fixed $\lambda, u, v, \bar{t} \equiv\left(t^{1}, t^{2}, \ldots, t^{\nu_{0}}\right)$, and $\bar{s} \equiv\left(s^{\nu_{0}+1}, s^{\nu_{0}+2}, \ldots, s^{\nu}\right)$, on $\mathbb{R}^{n}$ as follows:

$$
\begin{gathered}
\Phi_{i}(z, \lambda, v, \bar{t}, \bar{s})=f_{i}(z)-\lambda g_{i}(z)+\sum_{m \in J_{0}} v_{m} G_{j_{m}}\left(z, t^{m}\right)+\sum_{m \in K_{0}} v_{m} H_{k_{m}}\left(z, s^{m}\right), \quad i \in \underline{p}, \\
\Phi(z, \lambda, u, v, \bar{t}, \bar{s})=\sum_{i=1}^{p} u_{i}\left[f_{i}(z)-\lambda g_{i}(z)\right]+\sum_{m \in J_{0}} v_{m} G_{j_{m}}\left(z, t^{m}\right)+\sum_{m \in K_{0}} v_{m} H_{k_{m}}\left(z, s^{m}\right),
\end{gathered}
$$




$$
\Lambda_{\tau}(z, v, \bar{t}, \bar{s})=\sum_{m \in J_{\tau}} v_{m} G_{j_{m}}\left(z, t^{m}\right)+\sum_{m \in K_{\tau}} v_{m} H_{k_{m}}\left(z, s^{m}\right), \quad \tau \in \underline{M} .
$$

In the proofs of our sufficiency theorems, we shall make frequent use of the following auxiliary result which provides an alternative expression for the objective function of $(P)$.

Lemma 1. [7] For each $x \in X$,

$$
\varphi(x) \equiv \max _{1 \leq i \leq p} \frac{f_{i}(x)}{g_{i}(x)}=\max _{u \in U} \frac{\sum_{i=1}^{p} u_{i} f_{i}(x)}{\sum_{i=1}^{p} u_{i} g_{i}(x)} .
$$

Making use of the sets and functions defined above, we can now formulate our first collection of generalized sufficiency results for $(\mathrm{P})$ as follows.

Theorem 2.1. Let $x^{*} \in \mathbb{F}$, let $\lambda^{*}=\varphi\left(x^{*}\right)$, let the functions $f_{i}, g_{i}, i \in \underline{p}, z \rightarrow G_{j}(z, t)$, and $z \rightarrow H_{k}(z, s)$ be twice continuously differentiable at $x^{*}$ for all $t \in T_{j}$ and $s \in S_{k}, j \in \bar{q}, k \in \underline{r}$, and assume that there exist $u^{*} \in U$ and integers $\nu_{0}$ and $\nu$, with $0 \leq \nu_{0} \leq \nu \leq n+1$, such that there exist $\nu_{0}$ indices $j_{m}$, with $1 \leq j_{m} \leq q$, together with $\nu_{0}$ points $t^{m} \in \hat{T}_{j_{m}}\left(x^{*}\right), m \in \underline{\nu_{0}}, \nu-\nu_{0}$ indices $k_{m}$, with $1 \leq k_{m} \leq r$, together with $\nu-\nu_{0}$ points $s^{m} \in S_{k_{m}}, m \in \underline{\nu} \backslash \underline{\nu_{0}}$, and $\nu$ real numbers $v_{m}^{*}$ with $v_{m}^{*}>0$ for $m \in \underline{\nu_{0}}$, such that (1.1), (1.2) and (1.3) hold. Assume, further that any one of the following four sets of hypotheses is satisfied:

(a) (i) $z \rightarrow \Phi\left(z, \lambda^{*}, u^{*}, v^{*}, \bar{t}, \bar{s}\right)$ is prestrictly $(\mathcal{F}, \beta, \bar{\phi}, \bar{\rho}, \theta, m)$-quasisounivex at $x^{*}$, and $\bar{\phi}(a) \geq 0 \Rightarrow a \geq 0$;

(ii) for each $\tau \in \underline{M}, z \rightarrow \Lambda_{\tau}\left(z, v^{*}, \bar{t}, \bar{s}\right)$ is strictly $\left(\mathcal{F}, \beta, \tilde{\phi}_{\tau}, \tilde{\rho}_{\tau}, \theta, m\right)$-pseudosounivex at $x^{*}, \tilde{\phi}_{\tau}$ is increasing, and $\tilde{\phi}_{\tau}(0)=0$;

(iii) $\bar{\rho}+\sum_{\tau=1}^{M} \tilde{\rho}_{\tau} \geq 0$;

(b) (i) $z \rightarrow \Phi\left(z, \lambda^{*}, u^{*}, v^{*}, \bar{t}, \bar{s}\right)$ is $(\mathcal{F}, \beta, \bar{\phi}, \bar{\rho}, \theta, m)$-pseudosounivex at $x^{*}$, and $\bar{\phi}(a) \geq 0 \Rightarrow a \geq 0$;

(ii) for each $\tau \in \underline{M}, z \rightarrow \Lambda_{\tau}\left(z, v^{*}, \bar{t}, \bar{s}\right)$ is $\left(\mathcal{F}, \beta, \tilde{\phi}_{\tau}, \tilde{\rho}_{\tau}, \theta, m\right)$-quasisounivex at $x^{*}, \tilde{\phi}_{\tau}$ is increasing, and $\tilde{\phi}_{\tau}(0)=0$;

(iii) $\bar{\rho}+\sum_{\tau=1}^{M} \tilde{\rho}_{\tau} \geq 0$;

(c) (i) $z \rightarrow \Phi\left(z, \lambda^{*}, u^{*}, v^{*}, \bar{t}, \bar{s}\right)$ is prestrictly $(\mathcal{F}, \beta, \bar{\phi}, \bar{\rho}, \theta, m)$ - quasisounivex at $x^{*}$, and $\bar{\phi}(a) \geq 0 \Rightarrow a \geq 0$;

(ii) for each $\tau \in \underline{M}, z \rightarrow \Lambda_{\tau}\left(z, v^{*}, \bar{t}, \bar{s}\right)$ is $\left(\mathcal{F}, \beta, \tilde{\phi}_{\tau}, \tilde{\rho}_{\tau}, \theta, m\right)$ - quasisounivex at $x^{*}, \tilde{\phi}_{\tau}$ is increasing, and $\tilde{\phi}_{\tau}(0)=0$;

(iii) $\bar{\rho}+\sum_{\tau=1}^{M} \tilde{\rho}_{\tau}>0$;

(d) (i) $z \rightarrow \Phi\left(z, \lambda^{*}, u^{*}, v^{*}, \bar{t}, \bar{s}\right)$ is prestrictly $(\mathcal{F}, \beta, \bar{\phi}, \bar{\rho}, \theta, m)$ - quasisounivex at $x^{*}$, and $\bar{\phi}(a) \geq 0 \Rightarrow a \geq 0$;

(ii) for each $\tau \in \underline{M_{1}}, z \rightarrow \Lambda_{\tau}\left(z, v^{*}, \bar{t}, \bar{s}\right)$ is $\left(\mathcal{F}, \beta, \tilde{\phi}_{\tau}, \tilde{\rho}_{\tau}, \theta, m\right)$-quasisounivex at $x^{*}$, for each $\tau \in \underline{M_{2}} \neq$ $\emptyset, z \rightarrow \Lambda_{\tau}\left(z, v^{*}, \bar{t}, \bar{s}\right)$ is strictly $\left(\mathcal{F}, \beta, \tilde{\phi}_{\tau}, \tilde{\rho}_{\tau}, \theta, m\right)$-pseudosounivex at $x^{*}$, and for each $\tau \in \underline{M}, \overline{\tilde{\phi}_{\tau}}$ is increasing and $\tilde{\phi}_{\tau}(0)=0$, where $\left\{\underline{M_{1}}, \underline{M_{2}}\right\}$ is a partition of $\underline{M}$;

(iii) $\bar{\rho}+\sum_{\tau=1}^{M} \tilde{\rho}_{\tau} \geq 0$.

Then $x^{*}$ is an optimal solution of $(P)$.

Proof

Let $x$ be an arbitrary feasible solution of (P). 
(a) : In view of the positivity of $\beta\left(x, x^{*}\right)$ and sublinearity of $\mathcal{F}\left(x, x^{*} ; \cdot\right)$, it follows from (1.1) and (1.2) that

$$
\begin{gathered}
\mathcal{F}\left(x, x^{*} ; \beta\left(x, x^{*}\right)\left\{\sum_{i=1}^{p} u_{i}^{*}\left[\nabla f_{i}\left(x^{*}\right)-\lambda^{*} \nabla g_{i}\left(x^{*}\right)\right]+\sum_{m \in J_{0}} v_{m}^{*} \nabla G_{j_{m}}\left(x^{*}, t^{m}\right)+\right.\right. \\
\left.\left.\sum_{m \in K_{0}} v_{m}^{*} \nabla H_{k_{m}}\left(x^{*}, s^{m}\right)\right\}\right) \\
+\frac{1}{2}\left\langle z^{*},\left[\sum_{i=1}^{p} u_{i}^{*}\left[\nabla^{2} f_{i}\left(x^{*}\right)-\lambda^{*} \nabla^{2} g_{i}\left(x^{*}\right)\right]+\sum_{m \in J_{0}} v_{m}^{*} \nabla^{2} G_{j_{m}}\left(x^{*}, t^{m}\right)+\sum_{m \in K_{0}} v_{m}^{*} \nabla^{2} H_{k_{m}}\left(x^{*}, s^{m}\right)\right] z^{*}\right\rangle \\
+\mathcal{F}\left(x, x^{*} ; \beta\left(x, x^{*}\right) \sum_{\tau=1}^{M}\left[\sum_{m \in J_{\tau}} v_{m}^{*} \nabla G_{j_{m}}\left(x^{*}, t^{m}\right)+\sum_{m \in K_{\tau}} v_{m}^{*} \nabla H_{k_{m}}\left(x^{*}, s^{m}\right)\right]\right) \\
+\frac{1}{2}\left\langle z^{*}, \sum_{\tau=1}^{M}\left[\sum_{m \in J_{\tau}} v_{j}^{*} \nabla^{2} G_{j_{m}}\left(x^{*}, t^{m}\right)+\sum_{m \in K_{\tau}} v_{m}^{*} \nabla^{2} H_{k_{m}}\left(x^{*}, s^{m}\right)\right] z^{*}\right\rangle \geq 0 .
\end{gathered}
$$

Since $x, x^{*} \in \mathbb{F}$, for each $\tau \in \underline{M}$,

$$
\begin{aligned}
\Lambda_{\tau}\left(x, v^{*}, \bar{t}, \bar{s}\right) & =\sum_{m \in J_{\tau}} v_{m}^{*} G_{j_{m}}\left(x, t^{m}\right)+\sum_{m \in K_{\tau}} v_{m}^{*} H_{k_{m}}\left(x, s^{m}\right) \\
& \leq 0 \\
& =\sum_{m \in J_{\tau}} v_{m}^{*} G_{j_{m}}\left(x^{*}, t^{m}\right)+\sum_{m \in K_{\tau}} v_{m}^{*} H_{k_{m}}\left(x^{*}, s^{m}\right) \\
& =\Lambda_{\tau}\left(x^{*}, v^{*}, \bar{t}, \bar{s}\right),
\end{aligned}
$$

and so using the properties of the function $\tilde{\phi}_{\tau}$, we get

$$
\tilde{\phi}_{\tau}\left(\Lambda_{\tau}\left(x, v^{*}, \bar{t}, \bar{s}\right)-\Lambda_{\tau}\left(x^{*}, v^{*}, \bar{t}, \bar{s}\right)\right) \leq 0,
$$

which because of (ii) implies that

$$
\begin{aligned}
& \mathcal{F}\left(x, x^{*} ; \beta\left(x, x^{*}\right)[\right.\left.\left.\sum_{m \in J_{\tau}} v_{m}^{*} \nabla G_{j_{m}}\left(x^{*}, t^{m}\right)+\sum_{m \in K_{\tau}} v_{m}^{*} \nabla H_{k_{m}}\left(x^{*}, s^{m}\right)\right]\right) \\
&+\frac{1}{2}\left\langle z^{*},\left[\sum_{m \in J_{\tau}} v_{m}^{*} \nabla^{2} G_{j_{m}}\left(x^{*}, t^{m}\right)+\sum_{m \in K_{\tau}} v_{m}^{*} \nabla^{2} H_{k_{m}}\left(x^{*}, s^{m}\right)\right] z^{*}\right\rangle \\
&<-\tilde{\rho}_{\tau}\left(x, x^{*}\right)\left\|\theta\left(x, x^{*}\right)\right\|^{m} .
\end{aligned}
$$

Summing over $\tau$ and using the sublinearity of $\mathcal{F}\left(x, x^{*} ; \cdot\right)$, we obtain

$$
\begin{aligned}
\mathcal{F}\left(x, x^{*} ; \beta\left(x, x^{*}\right) \sum_{\tau=1}^{M}\left[\sum_{m \in J_{\tau}} v_{m}^{*} \nabla G_{j_{m}}\left(x^{*}, t^{m}\right)+\sum_{m \in K_{\tau}} v_{m}^{*} \nabla H_{k_{m}}\left(x^{*}, s^{m}\right)\right]\right) \\
+\frac{1}{2}\left\langle z^{*}, \sum_{\tau=1}^{M}\left[\sum_{m \in J_{\tau}} v_{m}^{*} \nabla^{2} G_{j_{m}}\left(x^{*}, t^{m}\right)+\sum_{m \in K_{\tau}} v_{m}^{*} \nabla^{2} H_{k_{m}}\left(x^{*}, s^{m}\right)\right] z^{*}\right\rangle \\
<-\sum_{\tau=1}^{M} \tilde{\rho}_{\tau}\left(x, x^{*}\right)\left\|\theta\left(x, x^{*}\right)\right\|^{m} .
\end{aligned}
$$


Combining (2.1), (2.2) and using (iii) we get

$$
\begin{aligned}
& \mathcal{F}\left(x, x^{*} ; \beta\left(x, x^{*}\right)\right.\left\{\sum_{i=1}^{p} u_{i}^{*}\left[\nabla f_{i}\left(x^{*}\right)-\lambda^{*} \nabla g_{i}\left(x^{*}\right)\right]+\sum_{m \in J_{0}} v_{m}^{*} \nabla G_{j_{m}}\left(x^{*}, t^{m}\right)\right. \\
&\left.\left.+\sum_{m \in K_{0}} v_{m}^{*} \nabla H_{k_{m}}\left(x^{*}, s^{m}\right)\right\}\right)+\frac{1}{2}\left\langle z^{*},\left[\sum_{i=1}^{p} u_{i}^{*}\left[\nabla^{2} f_{i}\left(x^{*}\right)-\lambda^{*} \nabla^{2} g_{i}\left(x^{*}\right)\right]+\sum_{m \in J_{0}} v_{m}^{*} \nabla^{2} G_{j_{m}}\left(x^{*}, t^{m}\right)\right.\right. \\
&\left.\left.+\sum_{m \in K_{0}} v_{m}^{*} \nabla^{2} H_{k_{m}}\left(x^{*}, s^{m}\right)\right] z^{*}\right\rangle>\sum_{\tau=1}^{M} \tilde{\rho}_{\tau}\left(x, x^{*}\right)\left\|\theta\left(x, x^{*}\right)\right\|^{m}>-\bar{\rho}\left(x, x^{*}\right) \|\left(\theta\left(x, x^{*}\right) \|^{m},\right.
\end{aligned}
$$

which by virtue of (i) implies that $\bar{\phi}\left(\Phi\left(x, \lambda^{*}, u^{*}, v^{*}, \bar{t}, \bar{s}\right)-\Phi\left(x^{*}, \lambda^{*}, u^{*}, v^{*}, \bar{t}, \bar{s}\right)\right) \geq 0$, which in light of further properties of $\bar{\phi}$ reduces to

$$
\Phi\left(x, \lambda^{*}, u^{*}, v^{*}, \bar{t}, \bar{s}\right) \geq \Phi\left(x^{*}, \lambda^{*}, u^{*}, v^{*}, \bar{t}, \bar{s}\right)=0,
$$

where the equality follows from (1.3) and the feasibility of $x^{*}$. Since $x, x^{*} \in \mathbb{F}$ and $v_{m}^{*}>0, m \in \underline{\nu_{0}}$, we have

$$
0 \leq \sum_{i=1}^{p} u_{i}^{*}\left[f_{i}(x)-\lambda^{*} g_{i}(x)\right] .
$$

Now using this inequality and Lemma 2.1, we obtain $\varphi\left(x^{*}\right) \leq \varphi(x)$. Since $x$ is arbitrary, we conclude that $x^{*}$ is an optimal solution to $(\mathrm{P})$.

(b): Based on the proof of part (a), we see that (ii) leads to the following inequality:

$$
\begin{array}{r}
\mathcal{F}\left(x, x^{*} ; \beta\left(x, x^{*}\right) \sum_{\tau=1}^{M}\left[\sum_{m \in J_{\tau}} v_{m}^{*} \nabla G_{j_{m}}\left(x^{*}, t^{m}\right)+\sum_{m \in K_{\tau}} v_{m}^{*} \nabla H_{k_{m}}\left(x^{*}, s^{m}\right)\right]\right) \\
+\frac{1}{2}\left\langle z^{*}, \sum_{\tau=1}^{M}\left[\sum_{m \in J_{\tau}} v_{j}^{*} \nabla^{2} G_{j_{m}}\left(x^{*}, t^{m}\right)+\sum_{m \in K_{\tau}} v_{m}^{*} \nabla^{2} H_{k_{m}}\left(x^{*}, s^{m}\right)\right] z^{*}\right\rangle \\
\leq-\sum_{\tau=1}^{M} \tilde{\rho}_{\tau}\left(x, x^{*}\right)\left\|\theta\left(x, x^{*}\right)\right\|^{m} .
\end{array}
$$

Now combining this inequality with (2.1) and using (iii), we obtain

$$
\begin{aligned}
\mathcal{F}\left(x, x^{*} ; \beta\left(x, x^{*}\right)\left\{\sum_{i=1}^{p} u_{i}^{*}\left[\nabla f_{i}\left(x^{*}\right)-\lambda^{*} \nabla g_{i}\left(x^{*}\right)\right]+\sum_{m \in J_{0}} v_{m}^{*} \nabla G_{j_{m}}\left(x^{*}, t^{m}\right)\right.\right. & \left.\left.+\sum_{m \in K_{0}} v_{m}^{*} \nabla H_{k_{m}}\left(x^{*}, s^{m}\right)\right\}\right) \\
+ & \frac{1}{2}\left\langle z^{*},\left[\sum_{i=1}^{p} u_{i}^{*}\left[\nabla^{2} f_{i}\left(x^{*}\right)-\lambda^{*} \nabla^{2} g_{i}\left(x^{*}\right)\right]+\sum_{m \in J_{0}} v_{m}^{*} \nabla^{2} G_{j_{m}}\left(x^{*}, t^{m}\right)\right.\right. \\
& \left.\left.+\sum_{m \in K_{0}} v_{m}^{*} \nabla^{2} H_{k_{m}}\left(x^{*}, s^{m}\right)\right] z^{*}\right\rangle \geq \sum_{\tau=1}^{M} \tilde{\rho}_{\tau}\left(x, x^{*}\right)\left\|\theta\left(x, x^{*}\right)\right\|^{m} \geq-\bar{\rho}\left(x, x^{*}\right)\left\|\theta\left(x, x^{*}\right)\right\|^{M},
\end{aligned}
$$

which applying (i) implies that $\bar{\phi}\left(\Phi\left(x, \lambda^{*}, u^{*}, v^{*}, \bar{t}, \bar{s}\right)-\Phi\left(x^{*}, \lambda^{*}, u^{*}, v^{*}, \bar{t}, \bar{s}\right)\right) \geq 0$. Thus, we conclude that $x^{*}$ is an optimal solution to $(P)$, while the proofs for (c) and (d) are similar to (a) and (b).

Making use of the sets and functions defined above, we can now formulate our first collection of generalized second-order parametric sufficient optimality results for $(P)$ as follows. 
Theorem 2.2. Let $x^{*} \in \mathbb{F}$, let $\lambda^{*}=\varphi\left(x^{*}\right)$, let the functions $f_{i}, g_{i}, i \in p, z \rightarrow G_{j}(z, t)$, and $z \rightarrow H_{k}(z, s)$ be twice continuously differentiable at $x^{*}$ for all $t \in T_{j}$ and $s \in S_{k}, j \in \bar{q}, k \in \underline{r}$, and assume that there exist $u^{*} \in U$ and integers $\nu_{0}$ and $\nu$, with $0 \leq \nu_{0} \leq \nu \leq n+1$, such that there exist $\nu_{0}$ indices $j_{m}$, with $1 \leq j_{m} \leq q$, together with $\nu_{0}$ points $t^{m} \in \hat{T}_{j_{m}}\left(x^{*}\right), m \in \underline{\nu_{0}}, \nu-\nu_{0}$ indices $k_{m}$, with $1 \leq k_{m} \leq r$, together with $\nu-\nu_{0}$ points $s^{m} \in S_{k_{m}}, m \in \underline{\nu} \backslash \underline{\nu_{0}}$, and $\nu$ real numbers $\overline{v_{m}^{*}}$ with $v_{m}^{*}>0$ for $m \in \underline{\nu_{0}}$, such that (1.1), (1.2) and (1.3) hold. Assume, further that any one of the following seven sets of hypotheses is satisfied:

(a) (i) for each $i \in I_{+} \equiv\left\{i \in \underline{p}: u_{i}^{*}>0\right\}, \xi \rightarrow \Phi_{i}\left(\xi, v^{*}, \lambda^{*}, \bar{t}, \bar{s}\right)$ is $\left(\mathcal{F}, \beta, \bar{\phi}_{i}, \bar{\rho}_{i}, \theta, m\right)$-pseudosounivex at $x^{*}, \bar{\phi}_{i}$ is strictly increasing, and $\bar{\phi}_{i}(0)=0$;

(ii) for each $t \in \underline{M}, \xi \rightarrow \Lambda_{t}\left(\xi, v^{*}, \bar{t}, \bar{s}\right)$ is $\left(\mathcal{F}, \beta, \tilde{\phi}_{t}, \tilde{\rho}_{t}, \theta, m\right)$-quasisounivex at $x^{*}$, $\tilde{\phi}_{t}$ is increasing, and $\tilde{\phi}_{t}(0)=0$

(iii) $\sum_{i \in I_{+}} u_{i}^{*} \bar{\rho}_{i}\left(x, x^{*}\right)+\sum_{t=1}^{M} \tilde{\rho}_{t}\left(x, x^{*}\right) \geq 0$ for all $x \in \mathbb{F}$;

(b) (i) for each $i \in I_{+}, \xi \rightarrow \Phi_{i}\left(\xi, v^{*}, \lambda^{*}, \bar{t}, \bar{s}\right)$ is prestrictly $\left(\mathcal{F}, \beta, \bar{\phi}_{i}, \bar{\rho}_{i}, \theta, m\right)$-quasisounivex at $x^{*}$, $\bar{\phi}_{i}$ is strictly increasing, and $\bar{\phi}_{i}(0)=0$;

(ii) for each $t \in \underline{M}, \xi \rightarrow \Lambda_{t}\left(\xi, v^{*}, \bar{t}, \bar{s}\right)$ is strictly $\left(\mathcal{F}, \beta, \tilde{\phi}_{t}, \tilde{\rho}_{t}, \theta\right)$-pseudosounivex at $x^{*}, \tilde{\phi}_{t}$ is increasing, and $\tilde{\phi}_{t}(0)=0$

(iii) $\sum_{i \in I_{+}} u_{i}^{*} \bar{\rho}_{i}\left(x, x^{*}\right)+\sum_{t=1}^{M} \tilde{\rho}_{t}\left(x, x^{*}\right) \geq 0$ for all $x \in \mathbb{F}$;

(c) (i) for each $i \in I_{+}, \xi \rightarrow \Phi_{i}\left(\xi, v^{*}, \lambda^{*}, \bar{t}, \bar{s}\right)$ is prestrictly $\left(\mathcal{F}, \beta, \bar{\phi}_{i}, \bar{\rho}_{i}, \theta, m\right)$-quasisounivex at $x^{*}$, $\bar{\phi}_{i}$ is strictly increasing, and $\bar{\phi}_{i}(0)=0$;

(ii) for each $t \in \underline{M}, \xi \rightarrow \Lambda_{t}\left(\xi, v^{*}, \bar{t}, \bar{s}\right)$ is $\left(\mathcal{F}, \beta, \tilde{\phi}_{t}, \tilde{\rho}_{t}, \theta, m\right)$-quasisounivex at $x^{*}, \tilde{\phi}_{t}$ is increasing, and $\tilde{\phi}_{t}(0)=0$

(iii) $\sum_{i \in I_{+}} u_{i}^{*} \bar{\rho}_{i}\left(x, x^{*}\right)+\sum_{t=1}^{M} \tilde{\rho}_{t}\left(x, x^{*}\right)>0$ for all $x \in \mathbb{F}$;

(d) (i) for each $i \in I_{1+}, \xi \rightarrow \Phi_{i}\left(\xi, v^{*}, \lambda^{*}, \bar{t}, \bar{s}\right)$ is $\left(\mathcal{F}, \beta, \bar{\phi}_{i}, \bar{\rho}_{i}, \theta, m\right)$-pseudosounivex at $x^{*}$, for each $i \in$ $I_{2+}, \xi \rightarrow \Phi_{i}\left(\xi, v^{*}, v^{*}, \lambda^{*}, \bar{t}, \bar{s}\right)$ is prestrictly $\left(\mathcal{F}, \beta, \bar{\phi}_{i}, \bar{\rho}_{i}, \theta, m\right)$-quasisounivex at $x^{*}$, and for each $i \in$ $I_{+}, \bar{\phi}_{i}$ is strictly increasing and $\bar{\phi}_{i}(0)=0$, where $\left\{I_{1+}, I_{2+}\right\}$ is a partition of $I_{+}$;

(ii) for each $t \in \underline{M}, \xi \rightarrow \Lambda_{t}\left(\xi, v^{*}, \bar{t}, \bar{s}\right)$ is strictly $\left(\mathcal{F}, \beta, \tilde{\phi}_{t}, \tilde{\rho}_{t}, \theta, m\right)$-pseudosounivex at $x^{*}, \tilde{\phi}_{t}$ is increasing, and $\tilde{\phi}_{t}(0)=0$

(iii) $\sum_{i \in I_{+}} u_{i}^{*} \bar{\rho}_{i}\left(x, x^{*}\right)+\sum_{t=1}^{M} \tilde{\rho}_{t}\left(x, x^{*}\right) \geq 0$ for all $x \in \mathbb{F}$;

(e) (i) for each $i \in I_{1+} \neq \emptyset, \xi \rightarrow \Phi_{i}\left(\xi, v^{*}, \lambda^{*}, \bar{t}, \bar{s}\right)$ is $\left(\mathcal{F}, \beta, \bar{\phi}_{i}, \bar{\rho}_{i}, \theta, m\right)$-pseudosounivex at $x^{*}$, for each $i \in I_{2+}, \xi \rightarrow \Phi_{i}\left(\xi, v^{*}, \lambda^{*}, \bar{t}, \bar{s}\right)$ is prestrictly $\left(\mathcal{F}, \beta, \bar{\phi}_{i}, \bar{\rho}_{i}, \theta, m\right)$-quasisounivex at $x^{*}$, and for each $i \in I_{+}, \bar{\phi}_{i}$ is strictly increasing and $\bar{\phi}_{i}(0)=0$, where $\left\{I_{1+}, I_{2+}\right\}$ is a partition of $I_{+}$;

(ii) for each $t \in \underline{M}, \xi \rightarrow \Lambda_{t}\left(\xi, v^{*}, v^{*}, \bar{t}, \bar{s}\right)$ is $\left(\mathcal{F}, \beta, \tilde{\phi}_{t}, \tilde{\rho}_{t}, \theta, m\right)$-quasisounivex at $x^{*}, \tilde{\phi}_{t}$ is increasing, and $\tilde{\phi}_{t}(0)=0$

(iii) $\sum_{i \in I_{+}} u_{i}^{*} \bar{\rho}_{i}\left(x, x^{*}\right)+\sum_{t=1}^{M} \tilde{\rho}_{t}\left(x, x^{*}\right) \geq 0$ for all $x \in \mathbb{F}$;

(f) (i) for each $i \in I_{+}, \xi \rightarrow \Phi_{i}\left(\xi, v^{*}, \lambda^{*}, \bar{t}, \bar{s}\right)$ is prestrictly $\left(\mathcal{F}, \beta, \bar{\phi}_{i}, \bar{\rho}_{i}, \theta, m\right)$-quasisounivex at $x^{*}, \bar{\phi}_{i}$ is strictly increasing, and $\bar{\phi}_{i}(0)=0$;

(ii) for each $t \in \underline{M}_{1} \neq \emptyset, \xi \rightarrow \Lambda_{t}\left(\xi, v^{*}, \bar{t}, \bar{s}\right)$ is strictly $\left(\mathcal{F}, \beta, \bar{\phi}_{t}, \tilde{\rho}_{t}, \theta, m\right)$-pseudosounivex at $x^{*}$, for each $t \in \underline{M}_{2}, \xi \rightarrow \Lambda_{t}\left(\xi, v^{*}, \bar{t}, \bar{s}\right)$ is $\left(\mathcal{F}, \beta, \tilde{\phi}_{t}, \tilde{\rho}_{t}, \theta, m\right)$-quasisounivex at $x^{*}$, and for each $t \in \underline{M}$, $\tilde{\phi}_{t}$ is increasing and $\tilde{\phi}_{t}(0)=0$, where $\left\{\underline{M}_{1}, \underline{M}_{2}\right\}$ is a partition of $\underline{M}$;

(iii) $\sum_{i \in I_{+}} u_{i}^{*} \bar{\rho}_{i}\left(x, x^{*}\right)+\sum_{t=1}^{M} \tilde{\rho}_{t}\left(x, x^{*}\right) \geq 0$ for all $x \in \mathbb{F}$;

(g) (i) for each $i \in I_{1+}, \xi \rightarrow \Phi_{i}\left(\xi, v^{*}, \lambda^{*}, \bar{t}, \bar{s}\right)$ is $\left(\mathcal{F}, \beta, \bar{\phi}_{i}, \bar{\rho}_{i}, \theta, m\right)$-pseudosounivex at $x^{*}$, for each $i \in$ $I_{2+}, \xi \rightarrow \Phi_{i}\left(\xi, v^{*}, \lambda^{*}, \bar{t}, \bar{s}\right)$ is prestrictly $\left(\mathcal{F}, \beta, \bar{\phi}_{i}, \bar{\rho}_{i}, \theta, m\right)$-quasisounivex at $x^{*}$, and for each $i \in$ $I_{+}, \bar{\phi}_{i}$ is strictly increasing and $\bar{\phi}_{i}(0)=0$, where $\left\{I_{1+}, I_{2+}\right\}$ is a partition of $I_{+}$; 
(ii) for each $t \in \underline{M}_{1}, \xi \rightarrow \Lambda_{t}\left(\xi, v^{*}, \bar{t}, \bar{s}\right)$ is strictly $\left(\mathcal{F}, \beta, \tilde{\phi}_{t}, \tilde{\rho}_{t}, \theta, m\right)$-pseudosounivex at $x_{\tilde{\phi_{t}}}^{*}$, for each $t \in$ $\underline{M}_{2}, \xi \rightarrow \Lambda_{t}\left(\xi, v^{*}, \bar{t}, \bar{s}\right)$ is $\left(\mathcal{F}, \beta, \tilde{\phi}_{t}, \tilde{\rho}_{t}, \theta, m\right)$-quasisounivex at $x^{*}$, and for each $t \in \underline{M}, \tilde{\phi}_{t}$ is increasing and $\tilde{\phi}_{t}(0)=0$, where $\left\{\underline{M}_{1}, \underline{M}_{2}\right\}$ is a partition of $\underline{M}$;

(iii) $\sum_{i \in I_{+}} u_{i}^{*} \bar{\rho}_{i}\left(x, x^{*}\right)+\sum_{t=1}^{M} \tilde{\rho}_{t}\left(x, x^{*}\right) \geq 0$ for all $x \in \mathbb{F}$;

(iv) $I_{1+} \neq \emptyset, \underline{M_{1}} \neq \emptyset$, or $\sum_{i \in I_{+}} u_{i}^{*} \bar{\rho}_{i}\left(x, x^{*}\right)+\sum_{t=1}^{M} \tilde{\rho}_{t}\left(x, x^{*}\right)>0$.

Then $x^{*}$ is an optimal solution of $(P)$.

Proof

(a): Assume that $x^{*}$ is not an optimal solution to $(P)$. Then there exists a feasible solution $\bar{x}$ of $(P)$ such that $\varphi(\bar{x})<\varphi\left(x^{*}\right)=\lambda^{*}$. Hence, we have

$$
f_{i}(\bar{x})-\lambda^{*} g_{i}(\bar{x})<0 \text { for each } i \in \underline{p} .
$$

Since $v^{*} \geq 0$, for each $i \in I_{+}$, we have

$$
\begin{aligned}
\Phi_{i}\left(\bar{x}, v^{*}, \lambda^{*}, \bar{t}, \bar{s}\right) & =f_{i}(\bar{x})-\lambda^{*} g_{i}(\bar{x})+\sum_{j \in J_{0}} v_{j}^{*} G_{j}\left(\bar{x}, t^{m}\right)+\sum_{k \in K_{0}} v_{k}^{*} H_{k}\left(\bar{x}, s^{m}\right) \\
& \left.\leq f_{i}(\bar{x})-\lambda^{*} g_{i}(\bar{x}) \quad \text { by the feasibility of } \bar{x}\right) \\
& <0 \\
& =f_{i}\left(x^{*}\right)-\lambda^{*} g_{i}\left(x^{*}\right)+\sum_{j \in J_{0}} v_{j}^{*} G_{j}\left(x^{*}, t^{m}\right)+\sum_{k \in K_{0}} w_{k}^{*} H_{k}\left(x^{*}, s^{m}\right)
\end{aligned}
$$

(by the feasibility of $x^{*}$ )

$$
=\Phi_{i}\left(x^{*}, v^{*}, w^{*}, \lambda^{*}, \bar{t}, \bar{s}\right) \text {, }
$$

and so using the properties of the function $\bar{\phi}_{i}$, we get

$$
\bar{\phi}_{i}\left(\Phi_{i}\left(\bar{x}, v^{*}, \lambda^{*}\right)-\Phi_{i}\left(x^{*}, v^{*}, \lambda^{*}\right)\right)<0,
$$

which in view of (i) implies that

$$
\begin{gathered}
\mathcal{F}\left(x, x^{*} ; \beta\left(x, x^{*}\right)\left[\nabla f_{i}\left(x^{*}\right)-\lambda^{*} \nabla g_{i}\left(x^{*}\right)+\sum_{m \in J_{0}} v_{m}^{*} \nabla G_{j_{m}}\left(x^{*}, t^{m}\right)+\sum_{m \in K_{0}} v_{m}^{*} \nabla H_{k_{m}}\left(x^{*}, s^{m}\right)\right]\right)+ \\
\frac{1}{2}\left\langle z^{*},\left[\nabla^{2} f_{i}\left(x^{*}\right)-\lambda^{*} \nabla^{2} g_{i}\left(x^{*}\right)+\sum_{m \in J_{0}} v_{m}^{*} \nabla^{2} G_{j}\left(x^{*}, t^{m}\right)+\sum_{m \in K_{0}} v_{m}^{*} \nabla^{2} H_{k_{m}}\left(x^{*}, s^{m}\right)\right] z^{*}\right\rangle \\
<-\bar{\rho}_{i}\left(x, x^{*}\right)\left\|\theta\left(\bar{x}, x^{*}\right)\right\|^{m} .
\end{gathered}
$$

Since $u^{*}>0, u_{i}^{*}=0$ for each $i \in \underline{p} \backslash I_{+}, \sum_{i=1}^{p} u_{i}^{*}=1$, and $\mathcal{F}\left(x, x^{*}, \cdot\right)$ is sublinear, the above inequalities yield

$$
\begin{array}{r}
\mathcal{F}\left(x, x^{*} ; \beta\left(x, x^{*}\right)\left\{\sum_{i=1}^{p} u_{i}^{*}\left[\nabla f_{i}\left(x^{*}\right)-\lambda^{*} \nabla g_{i}\left(x^{*}\right)\right]+\sum_{m \in J_{0}} v_{m}^{*} \nabla G_{j_{m}}\left(x^{*}, t^{m}\right)+\sum_{m \in K_{0}} v_{m}^{*} \nabla H_{k_{m}}\left(x^{*}, s^{m}\right)\right\}\right)+ \\
\frac{1}{2}\left\langle z^{*},\left\{\sum_{i=1}^{p} u_{i}^{*}\left[\nabla^{2} f_{i}\left(x^{*}\right)-\lambda^{*} \nabla^{2} g_{i}\left(x^{*}\right)\right]+\sum_{m \in J_{0}} v_{m}^{*} \nabla^{2} G_{j_{m}}\left(x^{*}, t^{m}\right)+\sum_{m \in K_{0}} v_{m}^{*} \nabla^{2} H_{k_{m}}\left(x^{*}, s^{m}\right)\right\} z^{*}\right\rangle \\
<-\sum_{i \in I_{+}} u_{i}^{*} \bar{\rho}_{i}\left(x, x^{*}\right)\left\|\theta\left(\bar{x}, x^{*}\right)\right\|^{m} .
\end{array}
$$


Proceeding as in the proof of Theorem 2.1, we see that our assumptions in (ii) lead to

$$
\begin{aligned}
\mathcal{F}\left(x, x^{*} ; \beta\left(x, x^{*}\right) \sum_{\tau=1}^{M}\left[\sum_{m \in J_{\tau}} v_{m}^{*} \nabla G_{j_{m}}\left(x^{*}, t^{m}\right)\right.\right. & \left.\left.+\sum_{m \in K_{\tau}} v_{m}^{*} \nabla H_{k_{m}}\left(x^{*}, s^{m}\right)\right]\right) \\
+ & \frac{1}{2}\left\langle z^{*}, \sum_{\tau=1}^{M}\left[\sum_{m \in J_{\tau}} v_{m}^{*} \nabla^{2} G_{j}\left(x^{*}, t^{m}\right)\right.\right. \\
& \left.\left.+\sum_{m \in K_{\tau}} v_{m}^{*} \nabla^{2} H_{k_{m}}\left(x^{*}, s^{m}\right)\right] z^{*}\right\rangle \leq-\sum_{\tau=1}^{M} \tilde{\rho}_{t}\left(x, x^{*}\right)\left\|\theta\left(\bar{x}, x^{*}\right)\right\|^{2},
\end{aligned}
$$

which when combined with (2.1) results in

$$
\begin{aligned}
\mathcal{F}\left(x, x^{*} ; \beta\left(x, x^{*}\right)\{\right. & \sum_{i=1}^{p} u_{i}^{*}\left[\nabla f_{i}\left(x^{*}\right)-\lambda^{*} \nabla g_{i}\left(x^{*}\right)\right]+\sum_{m \in J_{0}} v_{m}^{*} \nabla G_{j_{m}}\left(x^{*}, t^{m}\right) \\
+ & \left.\left.\sum_{m \in K_{0}} v_{m}^{*} \nabla H_{k_{m}}\left(x^{*}, s^{m}\right)\right\}\right)+\frac{1}{2}\left\langle z^{*},\left\{\sum_{i=1}^{p} u_{i}^{*}\left[\nabla^{2} f_{i}\left(x^{*}\right)-\lambda^{*} \nabla^{2} g_{i}\left(x^{*}\right)\right]\right.\right. \\
& \left.\left.+\sum_{m \in J_{0}} v_{m}^{*} \nabla^{2} G_{j_{m}}\left(x^{*}, t^{m}\right)+\sum_{m \in K_{0}} v_{m}^{*} \nabla^{2} H_{k_{m}}\left(x^{*}, s^{m}\right)\right\} z^{*}\right\rangle \geq \sum_{\tau=1}^{M} \tilde{\rho}_{\tau}\left(x, x^{*}\right)\left\|\theta\left(\bar{x}, x^{*}\right)\right\|^{2} .
\end{aligned}
$$

Based on (iii), this inequality contradicts based on our assumption (2.6). Hence, $x^{*}$ is an optimal solution to $(P)$. (b) - (g) : The proofs are similar to that of part (a).

In the following theorem, we construct a different partitioning method which appears to be general for formulating a general duality model for a multiobjective fractional programming problem, and then we present another collection of sufficient optimality results for $(P)$ which are different from those stated in Theorems 2.1 and 2.2. These results are formulated on a partition of $\underline{p}$ in addition to those of $\underline{\nu}_{0}$ and $\underline{\nu} \backslash \underline{\nu_{0}}$, and by placing appropriate generalized $(\mathcal{F}, \beta, \phi, \rho, \theta)$-univexity requirements on certain combinations of the functions $z \rightarrow u_{i}\left[f_{i}(z)-\lambda g_{i}(z)\right], z \rightarrow G_{j}(z, t)$, and $z \rightarrow H_{k}(z, s)$.

Let $\left\{I_{0}, I_{1}, \ldots, I_{d}\right\},\left\{J_{0}, J_{1}, \ldots, J_{e}\right\}$, and $\left\{K_{0}, K_{1}, \ldots, K_{e}\right\}$ be partitions of $p, \underline{\nu_{0}}$, and $\underline{\nu} \underline{\nu_{0}}$, respectively, such that $D=\{0,1,2, \ldots, d\} \subset E=\{0,1, \ldots, e\}$, and let the function $z \rightarrow \Pi_{\tau}(z, \bar{\lambda}, \bar{u}, v, \bar{t}, \bar{s}): \overline{\mathbb{R}}^{n} \rightarrow \mathbb{R}$ be defined, for fixed $\lambda, u, v, \bar{t} \equiv\left(t^{1}, t^{2}, \ldots, t^{\nu_{0}}\right)$, and $\bar{s} \equiv\left(s^{\nu_{0}+1}, s^{\nu_{0}+2}, \ldots, s^{\nu}\right)$, by

$$
\Pi_{\tau}(z, \lambda, u, v, \bar{t}, \bar{s})=\sum_{i \in I_{\tau}} u_{i}\left[f_{i}(z)-\lambda g_{i}(z)\right]+\sum_{m \in J_{\tau}} v_{m} G_{j_{m}}\left(x, t^{m}\right)
$$

$$
+\sum_{m \in K_{\tau}} v_{m} H_{k_{m}}\left(z, s^{m}\right), \quad \tau \in D
$$

Theorem 2.3. Let $x^{*} \in \mathbb{F}$, let $\lambda^{*}=\varphi\left(x^{*}\right)$, let the functions $f_{i}, g_{i}, i \in \underline{p}, z \rightarrow G_{j}(z, t)$, and $z \rightarrow H_{k}(z, s)$ be differentiable at $x^{*}$ for all $t \in T_{j}$ and $s \in S_{k}, j \in \underline{q}, k \in \underline{r}$, and assume that there exist $u^{*} \in U, u^{*}>0$, and integers $\nu_{0}$ and $\nu$, with $0 \leq \nu_{0} \leq \nu \leq n+1$, such that there exist $\nu_{0}$ indices $j_{m}$, with $1 \leq j_{m} \leq q$, together with $\nu_{0}$ points $t^{m} \in \hat{T}_{j_{m}}\left(x^{*}\right), m \in \underline{\nu_{0}}, \nu-\nu_{0}$ indices $k_{m}$, with $1 \leq k_{m} \leq r$, together with $\nu-\nu_{0}$ points $s^{m} \in S_{k_{m}}, m \in$ $\underline{\nu} \backslash \underline{\nu_{0}}$, and $\nu$ real numbers $v_{m}^{*}$ with $v_{m}^{*}>0$ for $m \in \underline{\nu_{0}}$, such that (1) and (2) hold. Assume, furthermore, that any one of the following seven sets of hypotheses is satisfied:

(a) (i) for each $\tau \in D, z \rightarrow \Pi_{\tau}\left(z, \lambda^{*}, u^{*}, v^{*}, \bar{t}, \bar{s}\right)$ is $\left(\mathcal{F}, \beta, \phi_{\tau}, \rho_{\tau}, \theta, m\right)$ - pseudosounivex at $x^{*}, \phi_{\tau}$ is increasing, and $\phi_{\tau}(0)=0$; 
(ii) for each $\tau \in E \backslash D, z \rightarrow \Lambda_{\tau}\left(z, v^{*}, \bar{t}, \bar{s}\right)$ is $\left(\mathcal{F}, \beta, \phi_{\tau}, \rho_{\tau}, m\right)$ - quasisounivex at $x^{*}, \phi_{\tau}$ is increasing, and $\phi_{\tau}(0)=0$;

(iii) $\sum_{\tau \in E} \rho_{\tau} \geq 0$;

(b) (i) for each $\tau \in D, z \rightarrow \Pi_{\tau}\left(z, \lambda^{*}, u^{*}, v^{*}, \bar{t}, \bar{s}\right)$ is prestrictly $\left(\mathcal{F}, \beta, \phi_{\tau}, \rho_{\tau}, \theta, m\right)$ - quasisounivex at $x^{*}, \phi_{\tau}$ is increasing, and $\phi_{\tau}(0)=0$;

(ii) for each $\tau \in E \backslash D, z \rightarrow \Lambda_{\tau}\left(z, v^{*}, \bar{t}, \bar{s}\right)$ is strictly $\left(\mathcal{F}, \beta, \phi_{\tau}, \rho_{\tau}, \theta, m\right)$ - pseudosounivex at $x^{*}$, $\phi_{\tau}$ is increasing, and $\phi_{\tau}(0)=0$;

(iii) $\sum_{\tau \in E} \rho_{\tau} \geq 0$;

(c) (i) for each $\tau \in D, z \rightarrow \Pi_{\tau}\left(z, \lambda^{*}, u^{*}, v^{*}, \bar{t}, \bar{s}\right)$ is prestrictly $\left(\mathcal{F}, \beta, \phi_{\tau}, \rho_{\tau}, \theta, m\right)$ - quasisounivex at $x^{*}, \phi_{\tau}$ is increasing, and $\phi_{\tau}(0)=0$;

(ii) for each $\tau \in E \backslash D, z \rightarrow \Lambda_{\tau}\left(z, v^{*}, \bar{t}, \bar{s}\right)$ is $\left(\mathcal{F}, \beta, \phi_{\tau}, \rho_{\tau}, \theta, m\right)$ - quasisounivex at $x^{*}, \phi_{\tau}$ is increasing, and $\phi_{\tau}(0)=0$

(iii) $\sum_{\tau \in E} \rho_{\tau}>0$;

(d) (i) for each $\tau \in D_{1}, z \rightarrow \Pi_{\tau}\left(z, \lambda^{*}, u^{*}, v^{*}, \bar{t}, \bar{s}\right)$ is $\left(\mathcal{F}, \beta, \phi_{\tau}, \rho_{\tau}, \theta, m\right)$ - pseudosounivex at $x^{*}$, for each $\tau \in D_{2}, z \rightarrow \Pi_{\tau}\left(z, \lambda^{*}, u^{*}, v^{*}, \bar{t}, \bar{s}\right)$ is prestrictly $\left(\mathcal{F}, \beta, \phi_{\tau}, \rho_{\tau}, \theta, m\right)$ - quasisounivex at $x^{*}$, and for each $\tau \in D, \phi_{\tau}$ is increasing and $\phi_{\tau}(0)=0$, where $\left\{D_{1}, D_{2}\right\}$ is a partition of $D$;

(ii) for each $\tau \in E \backslash D, z \rightarrow \Lambda_{\tau}\left(z, v^{*}, \bar{t}, \bar{s}\right)$ is strictly $\left(\mathcal{F}, \beta, \phi_{\tau}, \rho_{\tau}, \theta, m\right)$-pseudosounivex at $x^{*}, \phi_{\tau}$ is increasing, and $\phi_{\tau}(0)=0$;

(iii) $\sum_{\tau \in E} \rho_{\tau} \geq 0$;

(e) (i) for each $\tau \in D_{1} \neq \emptyset, z \rightarrow \Pi_{\tau}\left(z, \lambda^{*}, u^{*}, v^{*}, \bar{t}, \bar{s}\right)$ is $\left(\mathcal{F}, \beta, \phi_{\tau}, \rho_{\tau}, \theta, m\right)$ - pseudosounivex at $x^{*}$, for each $\tau \in D_{2}, z \rightarrow \Pi_{\tau}\left(z, \lambda^{*}, u^{*}, v^{*}, \bar{t}, \bar{s}\right)$ is prestrictly $\left(\mathcal{F}, \beta, \phi_{\tau}, \rho_{\tau}, \theta, m\right)$ - quasisounivex at $x^{*}$, and for each $\tau \in D, \phi_{\tau}$ is increasing and $\phi_{\tau}(0)=0$, where $\left\{D_{1}, D_{2}\right\}$ is a partition of $D$;

(ii) for each $\tau \in E \backslash D, z \rightarrow \Lambda_{\tau}\left(z, v^{*}, \bar{t}, \bar{s}\right)$ is $\left(\mathcal{F}, \beta, \phi_{\tau}, \rho_{\tau}, \theta, m\right)$ - quasisounivex at $x^{*}, \phi_{\tau}$ is increasing, and $\phi_{\tau}(0)=0$

(iii) $\sum_{\tau \in E} \rho_{\tau} \geq 0$;

(f) (i) for each $\tau \in D, z \rightarrow \Pi_{\tau}\left(z, \lambda^{*}, u^{*}, v^{*}, \bar{t}, \bar{s}\right)$ is prestrictly $\left(\mathcal{F}, \beta, \phi_{\tau}, \rho_{\tau}, \theta, m\right)$ - quasisounivex at $x^{*}, \phi_{\tau}$ is increasing, and $\phi_{\tau}(0)=0$;

(ii) for each $\tau \in(E \backslash D)_{1} \neq \emptyset, z \rightarrow \Lambda_{\tau}\left(z, v^{*}, \bar{t}, \bar{s}\right)$ is strictly $\left(\mathcal{F}, \beta, \phi_{\tau}, \rho_{\tau}, \theta, m\right)$ - pseudosounivex at $x^{*}$, for each $\tau \in(E \backslash D)_{2}, z \rightarrow \Lambda_{\tau}\left(z, v^{*}, \bar{t}, \bar{s}\right)$ is $\left(\mathcal{F}, \beta, \phi_{\tau}, \rho_{\tau}, \theta, m\right)$ - quasisounivex at $x^{*}$, and for each $\tau \in D, \phi_{\tau}$ is increasing and $\phi_{\tau}(0)=0$, where $\left\{(E \backslash D)_{1},(E \backslash D)_{2}\right\}$ is a partition of $E \backslash D$;

(iii) $\sum_{\tau \in E} \rho_{\tau} \geq 0$;

(g) (i) for each $\tau \in D_{1}, z \rightarrow \Pi_{\tau}\left(z, \lambda^{*}, u^{*}, v^{*}, \bar{t}, \bar{s}\right)$ is $\left(\mathcal{F}, \beta, \phi_{\tau}, \rho_{\tau}, \theta, m\right)$ - pseudosounivex at $x^{*}$, for each $\tau \in D_{2}, z \rightarrow \Pi_{\tau}\left(z, \lambda^{*}, u^{*}, v^{*}, \bar{t}, \bar{s}\right)$ is prestrictly $\left(\mathcal{F}, \beta, \phi_{\tau}, \rho_{\tau}, \theta, m\right)$ - quasisounivex at $x^{*}$, and for each $\tau \in D, \phi_{\tau}$ is increasing and $\phi_{\tau}(0)=0$, where $\left\{D_{1}, D_{2}\right\}$ is a partition of $D$;

(ii) for each $\tau \in(E \backslash D)_{1}, z \rightarrow \Lambda_{\tau}\left(z, v^{*}, \bar{t}, \bar{s}\right)$ is strictly $\left(\mathcal{F}, \beta, \phi_{\tau}, \rho_{\tau}, \theta, m\right)$ - pseudosounivex at $x^{*}$, for each $\tau \in(E \backslash D)_{2}, z \rightarrow \Lambda_{\tau}\left(z, v^{*}, \bar{t}, \bar{s}\right)$ is $\left(\mathcal{F}, \beta, \phi_{\tau}, \rho_{\tau}, \theta, m\right)$ - quasisounivex at $x^{*}$, and for each $\tau \in$ $D, \phi_{\tau}$ is increasing and $\phi_{\tau}(0)=0$, where $\left\{(E \backslash D)_{1},(E \backslash D)_{2}\right\}$ is a partition of $E \backslash D$;

(iii) $\sum_{\tau \in E} \rho_{\tau} \geq 0$;

(iv) $D_{1} \neq \emptyset,(E \backslash D)_{1} \neq \emptyset$, or $\sum_{\tau \in E} \rho_{\tau}>0$.

Then $x^{*}$ is an optimal solution of $(P)$.

Proof

(a): Assume that $x^{*}$ is not an optimal solution to $(P)$. Then based on the proof of Theorem 2.2, we arrive at the inequalities $f_{i}(\bar{x})-\lambda^{*} g_{i}(\bar{x})<0, i \in \underline{p}$, for some $\bar{x} \in \mathbb{F}$. Since $u^{*}>0$, we see that for each $\tau \in D$,

$$
\sum_{i \in I_{\tau}} u_{i}^{*}\left[f_{i}(\bar{x})-\lambda^{*} g_{i}(\bar{x})\right]<0 .
$$


Now using this inequality, we see that

$$
\begin{aligned}
\Pi_{\tau}\left(\bar{x}, \lambda^{*}, u^{*}, v^{*}, \bar{t}, \bar{s}\right) \\
=\sum_{i \in I_{\tau}} u_{i}^{*}\left[f_{i}(\bar{x})-\lambda^{*} g_{i}(\bar{x})\right]+\sum_{m \in J_{\tau}} v_{m}^{*} G_{j_{m}}\left(\bar{x}, t^{m}\right)+\sum_{m \in K_{\tau}} v_{m}^{*} H_{k_{m}}\left(\bar{x}, s^{m}\right) \\
\left.\leq \sum_{i \in I_{\tau}} u_{i}^{*}\left[f_{i}(\bar{x})-\lambda^{*} g_{i}(\bar{x})\right] \quad \text { by the feasibility of } \bar{x} \text { and positivity of } v_{m}^{*}, m \in \underline{\nu_{0}}\right) \\
<0 \\
=\sum_{i \in I_{\tau}} u_{i}^{*}\left[f_{i}\left(x^{*}\right)-\lambda^{*} g_{i}\left(x^{*}\right)\right]+\sum_{m \in J_{\tau}} v_{m}^{*} G_{j_{m}}\left(x^{*}, t^{m}\right)+\sum_{m \in K_{\tau}} v_{m}^{*} H_{k_{m}}\left(x^{*}, s^{m}\right) \\
\quad\left(\text { since }(1.3) \text { holds, } x^{*} \in \mathbb{F}, \text { and } t^{m} \in \hat{T}_{j_{m}}\left(x^{*}\right), m \in \underline{\nu_{0}}\right) \\
=\Pi_{\tau}\left(x^{*}, \lambda^{*}, u^{*}, v^{*}, \bar{t}, \bar{s}\right),
\end{aligned}
$$

and so using the properties of the functions $\phi_{\tau}, \tau \in D$, we get

$$
\phi_{\tau}\left(\Pi_{\tau}\left(\bar{x}, \lambda^{*}, u^{*}, v^{*}, \bar{t}, \bar{s}\right)-\Pi_{\tau}\left(x^{*}, \lambda^{*}, u^{*}, v^{*}, \bar{t}, \bar{s}\right)\right)<0,
$$

which in view of (i) implies that

$$
\begin{aligned}
\mathcal{F}\left(x, x^{*} ; \beta\left(x, x^{*}\right)\left\{\sum _ { i \in I _ { \tau } } u _ { i } ^ { * } \left[\nabla f_{i}\left(x^{*}\right)-\right.\right.\right. & \left.\lambda^{*} \nabla g_{i}\left(x^{*}\right)\right] \\
+ & +\sum_{m \in J_{\tau}} v_{m}^{*} \nabla G_{j_{m}}\left(x^{*}, t^{m}\right) \\
& \left.\left.\nabla H_{k_{m}}\left(x^{*}, s^{m}\right)\right\}\right) \\
+ & \frac{1}{2}\left\langle z^{*},\left\{\sum_{i \in I_{t}} u_{i}^{*}\left[\nabla^{2} f_{i}\left(x^{*}\right)-\lambda^{*} \nabla^{2} g_{i}\left(x^{*}\right)\right]+\sum_{j \in J_{t}} v_{j}^{*} \nabla^{2} G_{j}\left(x^{*}, t^{m}\right)\right.\right. \\
& \left.\left.+\sum_{k \in K_{t}} v_{k}^{*} \nabla^{2} H_{k}\left(x^{*}, s^{m}\right)\right\} z^{*}\right\rangle<-\rho_{t}\left(\bar{x}, x^{*}\right)\left\|\theta\left(\bar{x}, x^{*}\right)\right\|^{m} .
\end{aligned}
$$

Summing over $\tau \in D$ and using the sublinearity of $\mathcal{F}\left(x, x^{*} ; \cdot\right)$, we get

$$
\begin{aligned}
\mathcal{F}\left(x, x^{*} ; \beta\left(x, x^{*}\right)\left\{\sum _ { i = 1 } ^ { p } u _ { i } ^ { * } \left[\nabla f_{i}\left(x^{*}\right)-\right.\right.\right. & \left.\lambda \nabla g_{i}\left(x^{*}\right)\right]+\sum_{\tau \in D}\left[\sum_{m \in J_{\tau}} v_{m}^{*} \nabla G_{j_{m}}\left(x^{*}, t^{m}\right)\right. \\
& \left.\left.\left.+\sum_{m \in K_{\tau}} v_{m}^{*} \nabla H_{k_{m}}\left(x^{*}, s^{m}\right)\right]\right\}\right) \\
+ & \frac{1}{2}\left\langle z^{*},\left\{\sum_{i \in I_{\tau}} u_{i}^{*}\left[\nabla^{2} f_{i}\left(x^{*}\right)-\lambda^{*} \nabla^{2} g_{i}\left(x^{*}\right)\right]+\sum_{\tau \in D}\left[\sum_{m \in J_{\tau}} v_{m}^{*} \nabla^{2} G_{j_{m}}\left(x^{*}, t^{m}\right)\right.\right.\right. \\
& \left.\left.\left.+\sum_{m \in K_{\tau}} v_{m}^{*} \nabla^{2} H_{k_{m}}\left(x^{*}, s^{m}\right)\right]\right\} z^{*}\right\rangle<-\sum_{\tau \in D} \rho_{\tau}\left(\bar{x}, x^{*}\right)\left\|\theta\left(\bar{x}, x^{*}\right)\right\|^{m} .
\end{aligned}
$$

As shown in the proof of Theorem 2.2, for each $\tau \in E \backslash D, \Lambda_{\tau}\left(\bar{x}, v^{*}, \bar{t}, \bar{s}\right) \leq \Lambda_{\tau}\left(x^{*}, v^{*}, \bar{t}, \bar{s}\right)$, and so using the properties of $\phi_{\tau}, \tau \in E \backslash D$, we get the inequality $\phi_{\tau}\left(\Lambda_{\tau}\left(\bar{x}, v^{*}, \bar{t}, \bar{s}\right)-\Lambda_{\tau}\left(x^{*}, v^{*}, \bar{t}, \bar{s}\right)\right) \leq 0$, which in view of (ii) implies that

$$
\begin{aligned}
\mathcal{F}\left(x, x^{*} ; \beta\left(x, x^{*}\right)\left[\sum_{m \in J_{\tau}} v_{m}^{*} \nabla G_{j_{m}}\left(x^{*}, t^{m}\right)+\sum_{m \in K_{\tau}} v_{m}^{*} \nabla H_{k_{m}}\left(x^{*}, s^{m}\right)\right]\right) & \\
+\frac{1}{2}\left\langle z^{*},\left[\sum_{j \in J_{\tau}} v_{j}^{*} \nabla^{2} G_{j}\left(x^{*}, t^{m}\right)+\sum_{k \in K_{\tau}} v_{k}^{*} \nabla^{2} H_{k}\left(x^{*}, s^{m}\right)\right] z^{*}\right\rangle & \leq-\rho_{\tau}\left(\bar{x}, x^{*}\right)\left\|\theta\left(\bar{x}, x^{*}\right)\right\|^{m} .
\end{aligned}
$$


Summing over $\tau \in E \backslash D$ and using the sublinearity of $\mathcal{F}\left(x, x^{*} ; \cdot\right)$, we obtain

$$
\begin{array}{r}
\mathcal{F}\left(x, x^{*} ; \beta\left(x, x^{*}\right) \sum_{\tau \in E \backslash D}\left[\sum_{m \in J_{\tau}} v_{m}^{*} \nabla G_{j_{m}}\left(x^{*}, t^{m}\right)+\sum_{m \in K_{\tau}} v_{m}^{*} \nabla H_{k_{m}}\left(x^{*}, s^{m}\right)\right]\right) \\
+\frac{1}{2}\left\langle z^{*}, \sum_{\tau \in E \backslash D}\left[\sum_{j \in J_{\tau}} v_{j}^{*} \nabla^{2} G_{j}\left(x^{*}, t^{m}\right)+\sum_{k \in K_{\tau}} v_{k}^{*} \nabla^{2} H_{k}\left(x^{*}, s^{m}\right)\right] z^{*}\right\rangle \\
\leq-\sum_{\tau \in E \backslash D} \rho_{\tau}\left(\bar{x}, x^{*}\right)\left\|\theta\left(\bar{x}, x^{*}\right)\right\|^{m} .
\end{array}
$$

Now combining (2.8) and (2.9) and using (iii), we have

$$
\begin{gathered}
\mathcal{F}\left(x, x^{*} ; \beta\left(x, x^{*}\right)\left\{\sum_{i=1}^{p} u_{i}^{*}\left[\nabla f_{i}\left(x^{*}\right)-\lambda^{*} \nabla g_{i}\left(x^{*}\right)\right]+\sum_{m=1}^{\nu_{0}} v_{m}^{*} \nabla G_{j_{m}}\left(x^{*}, t^{m}\right)+\right.\right. \\
\left.\left.\sum_{m=\nu_{0}+1}^{\nu} v_{m}^{*} \nabla H_{k_{m}}\left(x^{*}, s^{m}\right)\right\}\right) \\
+\left\langle z^{*},\left[\sum_{i=1}^{p} u_{i}^{*}\left[\nabla^{2} f_{i}\left(x^{*}\right)-\lambda^{*} \nabla^{2} g_{i}\left(x^{*}\right)\right]+\sum_{m=1}^{\nu_{0}^{*}} v_{m}^{*} \nabla^{2} G_{j_{m}}\left(x^{*}, t^{m}\right)+\sum_{m=\nu_{0}^{*}+1}^{\nu^{*}} v_{m}^{*} \nabla^{2} H_{k_{m}}\left(x^{*}, s^{m}\right)\right] z^{*}\right\rangle \\
<-\sum_{\tau \in E} \rho_{\tau}\left(x, x^{*}\right)\left\|\theta\left(x, x^{*}\right)\right\|^{m} \leq 0,
\end{gathered}
$$

which contradicts (1.1) and (1.2) (since $\beta\left(x, x^{*}\right)>0$ and $\mathcal{F}\left(x, x^{*} ; 0\right)=0$ ). Therefore, $x^{*}$ is an optimal solution of $(\mathrm{P})$.

(b) - (g) : The proofs are similar to that of part (a).

The modified versions of Theorems 2.2 and 2.3 can be stated in a similar manner. Theorems $2.1-2.3$, and their modified versions encompass a large family of sufficient optimality conditions for $(P)$ whose members can easily be identified by appropriate choices of the partitioning sets $J_{\mu}, K_{\mu}, \mu \in \underline{m} \cup\{0\}$, and $I_{t}, t \in \underline{\ell} \cup\{0\}$. To this context, we state explicitly some important special cases of part (a) of Theorem 2.2 , for example, the following corollary.

Corollary 1. Let $x^{*} \in \mathbb{F}$, let $\lambda^{*}=\varphi\left(x^{*}\right)$, let the functions $f_{i}, g_{i}, i \in \underline{p}, z \rightarrow G_{j}(z, t)$, and $z \rightarrow H_{k}(z, s)$ be twice continuously differentiable at $x^{*}$ for all $t \in T_{j}$ and $s \in S_{k}, j \in \underline{q}, k \in \underline{r}$, and assume that there exist $u^{*} \in U$ and integers $\nu_{0}$ and $\nu$, with $0 \leq \nu_{0} \leq \nu \leq n+1$, such that there exist $\nu_{0}$ indices $j_{m}$, with $1 \leq j_{m} \leq q$, together with $\nu_{0}$ points $t^{m} \in \hat{T}_{j_{m}}\left(x^{*}\right), m \in \underline{\nu_{0}}, \nu-\nu_{0}$ indices $k_{m}$, with $1 \leq k_{m} \leq r$, together with $\nu-\nu_{0}$ points $s^{m} \in S_{k_{m}}, m \in \underline{\nu} \backslash \underline{\nu_{0}}$, and $\nu$ real numbers $v_{m}^{*}$ with $v_{m}^{*}>0$ for $m \in \underline{\nu_{0}}$, such that (1.1), (1.2) and (1.3) hold. Assume further that any one of the following ten sets of hypotheses is satisfied:

(a) (i) for each $i \in I_{+} \equiv\left\{i \in \underline{p}: u_{i}^{*}>0\right\}$, the function $\xi \rightarrow f_{i}(\xi)-\lambda^{*} g_{i}(\xi)$ is $\left(\mathcal{F}, \beta, \bar{\phi}_{i}, \bar{\rho}_{i}, \theta, m\right)$ pseudosounivex at $x^{*}, \bar{\phi}_{i}$ is strictly increasing, and $\bar{\phi}_{i}(0)=0$;

(ii) the function $\xi \rightarrow \sum_{j=1}^{q} v_{j}^{*} G_{j}(\xi, t)+\sum_{k=1}^{r} v_{k}^{*} H_{k}(\xi, s)$ is $(\mathcal{F}, \beta, \tilde{\phi}, \tilde{\rho}, \theta, m)$-quasisounivex at $x^{*}, \tilde{\phi}$ is increasing, and $\tilde{\phi}(0)=0$;

(iii) $\sum_{i \in I_{+}} u_{i}^{*} \bar{\rho}_{i}\left(x, x^{*}\right)+\tilde{\rho}\left(x, x^{*}\right) \geq 0$ for all $x \in \mathbb{F}$;

(b) (i) for each $i \in I_{+}$, the function $\xi \rightarrow f_{i}(\xi)-\lambda^{*} g_{i}(\xi)+\sum_{j=1}^{q} v_{j}^{*} G_{j}(\xi, t)+\sum_{k=1}^{r} v_{k}^{*} H_{k}(\xi, s)$ is $\left(\mathcal{F}, \beta, \bar{\phi}_{i}, \bar{\rho}_{i}, \theta, m\right)$-pseudosounivex at $x^{*}, \bar{\phi}_{i}$ is strictly increasing, and $\bar{\phi}_{i}(0)=0$;

(ii) $\sum_{i \in I_{+}} u_{i}^{*} \bar{\rho}_{i}\left(x, x^{*}\right) \geq 0$ for all $x \in \mathbb{F}$; 
(c) (i) for each $i \in I_{+}$, the function $\xi \rightarrow f_{i}(\xi)-\lambda^{*} g_{i}(\xi)+\sum_{j \in J_{0}} v_{j}^{*} G_{j}(\xi, t)+\sum_{k=1}^{r} v_{k}^{*} H_{k}(\xi, s)$ is $\left(\mathcal{F}, \beta, \bar{\phi}_{i}, \bar{\rho}_{i}, \theta, m\right)$-pseudosounivex at $x^{*}, \bar{\phi}_{i}$ is strictly increasing, and $\bar{\phi}_{i}(0)=0$;

(ii) for each $t \in \underline{M}, \xi \rightarrow \sum_{j \in J_{t}} v_{j}^{*} G_{j}(\xi, t)$ is $\left(\mathcal{F}, \beta, \bar{\phi}_{t}, \tilde{\rho}_{t}, \theta, m\right)$-quasisounivex at $x^{*}, \tilde{\phi}_{t}$ is increasing, and $\tilde{\phi}_{t}(0)=0$

(iii) $\sum_{i \in I_{+}} u_{i}^{*} \bar{\rho}_{i}\left(x, x^{*}\right)+\sum_{t=1}^{M} \tilde{\rho}_{t}\left(x, x^{*}\right) \geq 0$ for all $x \in \mathbb{F}$;

(d) (i) for each $i \in I_{+}$, the function $\xi \rightarrow f_{i}(\xi)-\lambda^{*} g_{i}(\xi)+\sum_{j=1}^{q} v_{j}^{*} G_{j}(\xi, t)+\sum_{k \in K_{0}} v_{k}^{*} H_{k}(\xi, s)$ is $\left(\mathcal{F}, \beta, \bar{\phi}_{i}, \bar{\rho}_{i}, \rho, \theta, m\right)$-pseudosounivex $x^{*}, \bar{\phi}_{i}$ is strictly increasing, and $\bar{\phi}_{i}(0)=0$;

(ii) for each $t \in \underline{M}, \xi \rightarrow \sum_{k \in K_{t}} v_{k}^{*} H_{k}(\xi, s)$ is $\left(\mathcal{F}, \beta, \tilde{\phi}_{t}, \tilde{\rho}_{t}, \theta, m\right)$-quasisounivex at $x^{*}, \tilde{\phi}_{t}$ is increasing, and $\tilde{\phi}_{t}(0)=0$;

(iii) $\sum_{i \in I_{+}} u_{i}^{*} \bar{\rho}_{i}\left(x, x^{*}\right)+\sum_{t=1}^{M} \tilde{\rho}_{t}\left(x, x^{*}\right) \geq 0$ for all $x \in \mathbb{F}$;

(e) (i) for each $i \in I_{+}$, the function $\xi \rightarrow f_{i}(\xi)-\lambda^{*} g_{i}(\xi)+\sum_{j=1}^{q} v_{j}^{*} G_{j}(\xi, t)$ is $\left(\mathcal{F}, \beta, \bar{\phi}_{i}, \bar{\rho}_{i}, \theta, m\right)$ pseudosounivex at $x^{*}, \bar{\phi}_{i}$ is strictly increasing, and $\bar{\phi}_{i}(0)=0$;

(ii) the function $\xi \rightarrow \sum_{k=1}^{r} v_{k}^{*} H_{k}(\xi, s)$ is $(\mathcal{F}, \beta, \tilde{\phi}, \tilde{\rho}, \theta, m)$-quasisounivex at $x^{*}, \tilde{\phi}$ is increasing, and $\tilde{\phi}(0)=0$;

(iii) $\sum_{i \in I_{+}} u_{i}^{*} \bar{\rho}_{i}\left(x, x^{*}\right)+\tilde{\rho}\left(x, x^{*}\right) \geq 0$ for all $x \in \mathbb{F}$;

(f) (i) for each $i \in I_{+}$, the function $\xi \rightarrow f_{i}(\xi)-\lambda^{*} g_{i}(\xi)+\sum_{k=1}^{r} v_{k}^{*} H_{k}(\xi, s)$ is $\left(\mathcal{F}, \beta, \bar{\phi}_{i}, \bar{\rho}_{i}, \theta, m\right)$ pseudosounivex at $x^{*}, \bar{\phi}_{i}$ is strictly increasing, and $\bar{\phi}_{i}(0)=0$;

(ii) the function $\xi \rightarrow \sum_{j=1}^{q} v_{j}^{*} G_{j}(\xi)$ is $(\mathcal{F}, \beta, \tilde{\phi}, \tilde{\rho}, \theta, m)$-quasisounivex at $x^{*}, \tilde{\phi}$ is increasing, and $\tilde{\phi}(0)=0$;

(iii) $\sum_{i \in I_{+}} u_{i}^{*} \bar{\rho}_{i}\left(x, x^{*}\right)+\tilde{\rho}\left(x, x^{*}\right) \geq 0$ for all $x \in \mathbb{F}$;

(g) (i) for each $i \in I_{+}$, the function $\xi \rightarrow f_{i}(\xi)-\lambda^{*} g_{i}(z)+\sum_{k=1}^{r} v_{k}^{*} H_{k}(\xi, s)$ is $\left(\mathcal{F}, \beta, \bar{\phi}_{i}, \bar{\rho}_{i}, \theta, m\right)$ pseudosounivex at $x^{*}, \bar{\phi}_{i}$ is strictly increasing, and $\bar{\phi}_{i}(0)=0$;

(ii) for each $j \in \underline{q}$, the function $\xi \rightarrow v_{j}^{*} G_{j}(\xi, t)$ is $\left(\mathcal{F}, \beta, \tilde{\phi}_{j}, \tilde{\rho}_{j}, \theta, m\right)$-quasisounivex at $x^{*}, \tilde{\phi}_{j}$ is increasing, and $\tilde{\phi}_{j}(0)=0$;

(iii) $\sum_{i \in I_{+}} u_{i}^{*} \bar{\rho}_{i}\left(x, x^{*}\right)+\sum_{j=1}^{q} \tilde{\rho}_{j}\left(x, x^{*}\right) \geq 0$ for all $x \in \mathbb{F}$;

(h) (i) for each $i \in I_{+}$, the function $\xi \rightarrow f_{i}(\xi)-\lambda^{*} g_{i}(\xi)+\sum_{j=1}^{q} v_{j}^{*} G_{j}(\xi, t)$ is $\left(\mathcal{F}, \beta, \bar{\phi}_{i}, \bar{\rho}_{i}, \theta, m\right)$ pseudosounivex at $x^{*}, \bar{\phi}_{i}$ is strictly increasing, and $\bar{\phi}_{i}(0)=0$;

(ii) for each $k \in \underline{r}, \xi \rightarrow w_{k}^{*} H_{k}(\xi, s)$ is $\left(\mathcal{F}, \beta, \tilde{\phi}_{k}, \tilde{\rho}_{k}, \theta, m\right)$-quasisounivex at $x^{*}$ and $\tilde{\phi}_{k}(0)=0$;

(iii) $\sum_{i \in I_{+}} u_{i}^{*} \bar{\rho}_{i}\left(x, x^{*}\right)+\sum_{k=1}^{r} \tilde{\rho}_{k}\left(x, x^{*}\right) \geq 0$ for all $x \in \mathbb{F}$;

(i) (i) for each $i \in I_{+}$, the function $\xi \rightarrow f_{i}(\xi)-\lambda^{*} g_{i}(\xi)+\sum_{j \in J_{0}} v_{j}^{*} G_{j}(\xi, t)+\sum_{k=1}^{r} v_{k}^{*} H_{k}(\xi, s)$ is $\left(\mathcal{F}, \beta, \bar{\phi}_{i}, \bar{\rho}_{i}, \theta, m\right)$-pseudosounivex at $x^{*}, \bar{\phi}_{i}$ is strictly increasing, and $\bar{\phi}_{i}(0)=0$;

(ii) the function $\xi \rightarrow \sum_{j \in J_{1}} v_{j}^{*} G_{j}(\xi, t)$ is $(\mathcal{F}, \beta, \tilde{\phi}, \tilde{\rho}, \theta, m)$-quasisounivex at $x^{*}, \tilde{\phi}$ is increasing, and $\tilde{\phi}(0)=0 ;$

(iii) $\sum_{i \in I_{+}} u_{i}^{*} \bar{\rho}_{i}\left(x, x^{*}\right)+\tilde{\rho}\left(x, x^{*}\right) \geq 0$ for all $x \in \mathbb{F}$;

(j) (i) for each $i \in I_{+}, \xi \rightarrow f_{i}(\xi)-\lambda^{*} g_{i}(\xi)+\sum_{j=1}^{q} v_{j}^{*} G_{j}(\xi, t)+\sum_{k \in K_{0}} v_{k}^{*} H_{k}(\xi, s)$ is $\left(\mathcal{F}, \beta, \bar{\phi}_{i}, \bar{\rho}_{i}, \theta, m\right)$ pseudosounivex at $x^{*}, \bar{\phi}_{i}$ is strictly increasing, and $\bar{\phi}_{i}(0)=0$;

(ii) the function $\xi \rightarrow \sum_{k \in K_{1}} v_{k}^{*} H_{k}(\xi, s)$ is $(\mathcal{F}, \beta, \tilde{\phi}, \tilde{\rho}, \theta, m)$-quasisounivex at $x^{*}, \tilde{\phi}$ is increasing, and $\tilde{\phi}(0)=0 ;$

(iii) $\sum_{i \in I_{+}} u_{i}^{*} \bar{\rho}_{i}\left(x, x^{*}\right)+\tilde{\rho}\left(x, x^{*}\right) \geq 0$ for all $x \in \mathbb{F}$.

Then $x^{*}$ is an optimal solution of $(P)$.

Proof

In general proofs follow based on appropriate manipulations, while comparing parts (f) and (g) of the above 
corollary, we observe that they represent two extreme cases with respect to the $(\mathcal{F}, \beta, \phi, \rho, \theta, m)$-quasisounivexity assumptions in the sense that in (f) all the functions $\xi \rightarrow v_{j}^{*} G_{j}(\xi)$, are lumped together, whereas in (g) separate $(\mathcal{F}, \beta, \phi, \rho, \theta, m)$-quasisounivexity conditions are imposed on the individual functions.

\section{Concluding Remarks}

We have established several sets of generalized second-order parametric sufficient optimality criteria for a semiinfinite discrete minmax fractional programming problem using a variety of generalized $(\mathcal{F}, \beta, \phi, \rho, \theta, m)$ sounivexity assumptions. These optimality results can be applied to constructing various duality models as well as for developing new algorithms for the numerical solution of minmax fractional programming problems. The results investigated in this communication seem to be applicable to further generalizations and challenging applications to higher order exponential type $(\mathcal{F}, \beta, \phi, \rho, \theta, m)$-sounivexities based on the recently introduced notion of the Hanson-Antczak type invexities by Zalmai [8].

\section{REFERENCES}

1. W. Dinkelbach, On nonlinear fractional programming, Management Sci. 13 (1967), 492 - 498.

2. M. A. Hanson, Second order invexity and duality in mathematical programming, Opsearch 30 (1993), 313 - 320.

3. B. Mond and T. Weir, Generalized concavity and duality, Generalized Concavity in Optimization and Economics (S. Schaible and W. T. Ziemba, eds.), Academic Press, New York, 1981, pp. 263 - 279.

4. R. U. Verma and G. J. Zalmai, Second-order parametric optimality conditions in semiinfinite discrete minmax fractional programming based on generalized $(\phi, \eta, \rho, \theta, \tilde{m})$-sonvexities, Transactions on Mathematical Programming and Applications 4 (1) (2016), 62 - 84.

5. R. U. Verma and G. J. Zalmai, Generalized higher order $(\phi, \eta, \zeta, \rho, \theta, m)$-invexities and parametric optimality conditions for discrete minmax fractional programming, Transactions on Mathematical Programming and Applications 4 (1) (2016), 85 - 101.

6. G. J. Zalmai, Optimality conditions and duality for constrained measurable subset selection problems with minmax objective functions, Optimization 20 (1989), 377 - 395.

7. G. J. Zalmai, Generalized second-order $(F, \beta, \phi, \rho, \theta)$-univex functions and parametric duality models in semiinfinite discrete minmax fractional programming, Adv. Nonlin. Var. Ineq. 15 (2012), 63 - 91.

8. G. J. Zalmai, Hanson-Antczak-type generalized $(\alpha, \beta, \gamma, \xi, \eta, \rho, \theta)$-V-invex functions in semiinfinite multiobjective fractional programming, Part I: Sufficient efficiency conditions, Advances in Nonlinear Variational Inequalities 16 (1) (2013), 91 - 114.

9. G. J. Zalmai and Qinghong Zhang, Generalized $(F, \beta, \phi, \rho, \theta)$-univex functions and global parametric sufficient optimality conditions in semiinfinite discrete minmax fractional programming, PanAmerican Math. J. 17 (2007), 1 - 26. 